\title{
Appareil
}

$22 \mid 2020$

Une philosophie de l'appareil

\section{Dé-crypte numérique}

\section{Germain Roesz}

\section{(2) OpenEdition}

Journals

Édition électronique

URL : http://journals.openedition.org/appareil/3603

DOI : 10.4000/appareil.3603

ISSN : 2101-0714

Éditeur

MSH Paris Nord

\section{Référence électronique}

Germain Roesz, « Dé-crypte numérique », Appareil [En ligne], 22 | 2020, mis en ligne le 21 décembre 2020, consulté le 26 mars 2021. URL : http://journals.openedition.org/appareil/3603 ; DOI : https:// doi.org/10.4000/appareil.3603

Ce document a été généré automatiquement le 26 mars 2021.

\section{(c) (i) (2) $\Theta$}

Appareil est mis à disposition selon les termes de la Licence Creative Commons Attribution - Pas d'Utilisation Commerciale - Pas de Modification 4.0 International. 


\section{Dé-crypte numérique}

\section{Germain Roesz}

1 Dans le numéro trimestriel d'Art Press 2 de mai-juin-juillet 2013 consacré à l'art dans le tout numérique, Jean-Louis Déotte signe un article qui s'intitule:«La crypte numérique ». Dans cette contribution, le philosophe reprend les points de sa théorie des appareils et, dans le même temps, fournit une interrogation (voire des intuitions) sur ce que peut le numérique, sur ce qu'il est, sur ce qu'il peut devenir.

2 Il donne, pour commencer, une définition de ce qu'il appelle "appareils»: «ces dispositifs techniques qui, comme la camera obscura inventée par les arabes, puis beaucoup plus tard la perspective linéaire et enfin la photographie, le cinéma - mais aussi les dispositifs institutionnels comme le musée ou le concert public. Ces appareils donnent leurs caractéristiques aux arts, époque après époque. Et les révolutions qui font passer d'une ère de l'écriture à une autre sont encore plus fondamentales, plus infrastructurelles que l'invention de telle ou telle famille d'appareils. C'est chaque fois la réalité qui s'en trouve remodelée et non seulement sa représentation ${ }^{1}$. »

3 Si la perspective est un appareil, la surface d'inscription sur laquelle et par laquelle elle se donne à voir et à comprendre n'est alors que le signe de la représentation soumise à l'appareil. La perspective, et il faut l'entendre sémantiquement, est un projet qui fait sens dans le temps. C'est bien entendu la question fondamentale qui se tient entre l'existant, ce que je vois, mais avant tout ce comment je me rends conscient, et comment je le fais savoir.

4 C'est certainement dans ce sens que Jean-Louis Déotte dit que ce qui se joue aujourd'hui, avec le numérique, "c'est l'abandon du monde de la représentation, et donc du projet, pour celui du programme ». Cette hypothèse s'inscrit dans la recherche menée longuement sur la conséquence de l'appareil et des appareils. Un projet, c'est l'élaboration "d'idées qui servent de boussole à la production future du monde, à l'action politique (idéologies)». Jean-Louis Déotte rappelle, et à juste titre, que «la modernité peut se caractériser par cette capacité, collective et subjective à faire des projets ». Il ajoute, et c'est fondamental, qu'avec un programme, cela n'est pas possible, car un programme "quelle que soit la réalité qu'il détermine, doit nécessairement se réaliser ». Ce que j'appelle Hyperréalisme numérique, le philosophe l'appelle « Réalisme 
intégral » où « la différence essentielle (...) tient entre être (ici-bas) et devoir-être (audelà) qui s'efface ».

5 L'attention est alors portée sur ce qui, non seulement modifie notre rapport au monde (hier, aujourd'hui, demain), mais sur l'hypothèse d'un devenir possible dans lequel et pour lequel j'injecterai volontiers une dose d'éthique (donc un avenir possible). Je sais bien que derrière un programme, il y a un humain; quoiqu'avec le projet de l'intelligence artificielle on doit penser que le programme peut être fabriqué par un programme. On peut ainsi s'inquiéter que de programme en programme, auto-générés (à l'infini), la possibilité d'arrêter le processus soit impossible.

6 Je veux toutefois revenir au terme de Réalisme intégral, qu'il faut peut-être comprendre de la manière suivante : qu'il n'est en soi que la réalité numérique. Ce que l'appareil observe du réel, il le calcule. Ce qu'il rend de ce calcul ne serait donc pas une présentation seconde, mais bien la présentation même du programme qui ferait croire à la réalité extérieure (à notre regard, à notre compréhension). C'est en ce sens qu'on peut comprendre le dit du philosophe : «l'écriture numérique [...] ne part que d'ellemême pour ne rencontrer que ses propres produits ». On peut penser aussi que j'appuie à un endroit précis, comme artiste peintre, bien sûr, qui tiendrait à protéger et à conserver sa propre liberté (pourquoi ne le ferait-il pas, d'ailleurs ?). Je sais bien, et le travail de Jean-Louis Déotte l'a montré, que, quelle que soit la production plastique à laquelle nous procédons, elle participe des appareils, mais les artistes ont la possibilité d'en échapper, en les contestant, en les omettant, en leur opposant des formes qui sortiraient de la norme. Cette opposition (qui parfois prend la forme de l'outrance) est bien entendu contenue dans ce qu'on appellera l'époque, qui peut la recevoir ou la combattre (qui peut accepter cette opposition, ou la refuser).

7 La question reste de voir si, avec le numérique, une mise en critique de celui-ci est possible. C'est par exemple ce que pense d'une certaine manière Dominique Moulon pour l'artiste Julien Levesque, qu'il appelle l'artiste de l'ubiquité. "Son dispositif intitulé Street Views Patchwork (2009) est entièrement automatique. Les quatre bandes horizontales qui recomposent un paysage global témoignent de multiples temporalités où l'Utah côtoie la Bretagne, la Finlande et le Japon. Pourtant les paysages qui se succèdent sont plausibles. [...] Au regard de l'habile détournement proposé par cet artiste français, nous sommes à la fois hier et aujourd'hui, ici et là-bas ${ }^{2} »$. Pour moi, il ne s'agit que d'une procédure imaginaire, possible grâce au numérique, mais déjà existante grâce aux ciseaux. Il s'agit encore d'un collage, sauf que le fait de supprimer la temporalité, l'espace, de faire croire au plausible (qui est alors un faux), renforce l'idée de Réalité intégrale (c'est-à-dire de réalité numérique en soi, partant de soi et renvoyant à soi). Il s'agit de la suppression des repères pour un paysage globalisé, c'està-dire unifié et " partagé " par tous, comme le dit l'auteur, à quoi il faut ajouter que c'est aussi le reflet d'un capitalisme total, invisible, ce qui confirme l'hypothèse de Déotte, « de l'effacement de la différence essentielle [...] qui se tient entre être (ici-bas) et devoir-être (au-delà) ».

8 Le tenant du numérique (comme art aussi, ce qui me paraît plus que problématique) ne considère précisément pas ce que cela fait à la disparition de rapports sensibles dans l'histoire passée et à venir. Cette occultation tient-elle à la croyance béate d'un monde nouveau qui remplacerait positivement l'ancien? D'autre part, la technique remplacet-elle l'évènement sensible subjectif ? Précisons que ce « subjectif » est pensé dans des 
rapports esthétiques et politiques, c'est-à-dire qu'il s'inscrit dans un appareil que l'auteur ignore peut-être.

9 Peut-être doit-on comprendre en ce sens la remarque de Jean-Louis Déotte : [...] il y a désormais la possibilité d'une production sans reste de la réalité, à partir du langage le plus formel, le plus vide de sens, le plus logico-mathématique. Cette production où il n'y a plus de différence entre le sensible et l'intelligible, entre le phénomène et la chose en soi, est incompréhensible pour l'esthétique en général et celle de Rancière en particulier ».

Notons cependant que la proposition numérique, quand elle se donne dans une forme (et dans le laisser-visible du passé), produit parfaitement du sensible puisque ce dernier est, à ce moment précis, un donné critique, comparatif et qui remarque les différents temps de l'histoire. Lorsque Jean-Louis Déotte pense à Metropol Parasol (place de la Encarnación à Séville) il précise que « la différence des temps a été préservée puisque l'ensemble des gigantesques parasols s'élève au-dessus des ruines romaines ». Il s'agit donc bien d'une porosité ( du rapport forme/contenu ») qui pourrait combattre le tout numérique ne produisant que lui-même. Le numérique, donc, fait forme, qui elle-même fait le corps sensible qui échappe au numérique.

11 Chaque point du texte de Jean-Louis Déotte mérite qu'on s'y arrête : par exemple, l'idée d'une rumeur numérique qui engloberait tout artiste dans le flux numérique. Cette immersion peut fonder une transformation des ressentis et des expressions. Pourtant, l'exemple de Kurt Schwitters (le Merzbau que le philosophe appelle la grotte, la cavité intime), le conduit à penser que l'art numérique ne pourrait exister que si les artistes devenaient des cryptophores ${ }^{3}$. Alors, poussons l'idée en pensant que la pixellisation n'est qu'une forme mathématico-logique du pigment originel, de ce qui se donne à voir dans les grottes ornées. Il y avait certes le souffle du corps, de la bouche qui parle le pigment, et on doit se questionner alors sur la façon dont l'artiste peut souffler la binarité numérique. Il peut le faire par l'imaginaire, en le maintenant dans une structure inventive, prospective, spéculative et sûrement en ayant intégré les socles les plus lointains de la construction de notre humanité.

12 Rappelons qu'en 1988, Edmond Couchot et Michel Bret, en collaboration avec MarieHélène Tramus, réalisaient une des premières œuvres numériques dites interactives, Je sème à tout vent $t^{4}$. L'œuvre fait explicitement référence au logo des éditions Larousse (qui depuis 1876 représente une semeuse soufflant une fleur de pissenlit afin d'en disperser les ombelles). On doit y voir la symbolisation de l'émancipation de l'homme grâce à la connaissance et à sa diffusion. Cette référence revendiquée par Edmond Couchot et Michel Bret met Pissenlits dans un moment charnière entre la culture analogique et la culture numérique, c'est-à-dire dans une articulation entre arts et sciences. L'œuvre se fonde, se transforme en réaction au souffle des spectateurs. Prouesse technique et dimension poétique transposent à l'image (pour l'image) cette réalité que nous pratiquions souvent dans l'enfance. À propos de cette œuvre Pissenlits ${ }^{5}$, on parlait à l'époque de vie artificielle prenant forme grâce au souffle réel. Sans creuser davantage, cette œuvre ouvrait le champ des possibles où le fonctionnement numérique autonome pouvait peut-être se dispenser du corps (mais le convoquait pourtant par intuition). Le fait qu'il s'agit d'une origine, joint à l'hypothèse de Jean-Louis Déotte d'un devenir cryptophore des artistes, on peut pousser encore plus loin et dire que la pixellisation est une sorte de pigment, que l'ombelle est la métaphore de ce pigment numérique qui ne trouve sens que dans la présence (encore) du souffleur. Ce dernier est un inspirateur 
qui, dans cette œuvre d'origine, comprend bien que le corps sera l'enjeu de ce qui pourrait être un art numérique. La compréhension d'une caverne intime, la fondation même de cette crypte (qui alors ne serait pas la seule imposition immersive du numérique dans nos vie) donnerait encore sens à nos déambulations poétiques, nos spéculations utopiques, nos découvertes non arrêtées par un mur de vérités technologiques.

Schmerz est peut-être à l'origine de Merz, tout comme Kommerz ${ }^{6}$. Cette douleur est pourtant nécessaire pour pouvoir commercer et se souvenir que la mémoire fait défaut, que le chef d'œuvre de Schwitters fut détruit et que la bombe que nous fabriquons est parfois au cœur des œuvres. Une cavité protectrice, ombilicale, est sûrement nécessaire pour que nous remettions du sens dans le flux incessant. Immersion certes, mais volontaire, qui distingue le vrai du faux.

Reste à considérer le lieu même de la crypte, ou ce qui la constitue. Dans la vérité en peinture, Jacques Derrida ouvre une piste.

7 janvier 1978

Quand la date elle-même devient le lieu d'une crypte, quand elle en tient lieu.

Sauront-ils jamais pourquoi j'inscris ceci à telle date?

Coup de dé7.

Dater le moment de conscience, intérioriser le sens de ce qui se joue, jusqu'au numérique, peut donc faire crypte. Jouer le désir d'un coup. Inattendu.

Cependant, quelque chose, du vivre le plus profond, échappera toujours.

On a dit aussi le date (la chose donnée, le datum). Il y a la date d'aujourd'hui, dont ils ne sauront jamais rien de ce qui y fut donné à vivre - et retiré.

La date elle-même tiendra lieu de crypte, la seule qui reste, fors le cœur ${ }^{8}$.

La crypte contient et éloigne dans le même temps ce qui la définit, la fait vivre, l'engage. Dans l'exécution, il y a de la présence, dans le résultat, il y a mémoire sans traces'.

Dater se dit en droit, du lieu d'écriture ou de signature pour l'engagement, le contrat, la missive, le testament. Qui saura jamais d'où je date ceci, aujourd'hui même? Il y a le don (es gibt) et il y a aussi ce qu'aujourd'hui je n'aurai pas pu donner. Et que donc je veux garder mieux que jamais ${ }^{10}$.

La crypte qu'on doit appeler de ses vœux n'a pas d'assignation au départ. Elle est le lieu lui-même (de la réception), elle est constituée d'un autre lieu (dont elle dit ou dira peu de choses), elle est le signe de la conscience de son antre, de son intime, de ce qu'elle peut porter à l'extérieur. Le numérique est alors non pas le liquide amniotique de tout devenir, mais une forme parmi les formes qui peut fonder du sens autant avec l'histoire qu'avec l'avenir. Il faut évidemment un liant pour le pigment, afin que le numérique ne soit pas le daté.

\section{BIBLIOGRAPHIE}

Déotte Jean-Louis, « L'appareil artistique de Schwitters », Appareil, n 9, 2012.

Déotte Jean-Louis, « La crypte numérique », Art Press 2, n 29, 2013, p. 36-43. 
Moulon Dominique, « Les pratiques numériques d'un art contemporain », Art Press 2, n 29, 2013, p. 19.

Derrida Jacques, La vérité en peinture, Paris, Champs Flammarion, 1978.

\section{NOTES}

1. Jean-Louis Déotte, « La crypte numérique », Art Press 2, n² 29, 2013, p. 36-43.

2. Dominique Moulon, «Les pratiques numériques d'un art contemporain », Art Press $2, \mathrm{n}^{\circ} 29$, 2013, p. 19.

3. Voir Jean-Louis Déotte, « L'appareil artistique de Schwitters », Appareil, nº 9, 2012.

4. Elle sera renommée en 2005 Pissenlits.

5. Le pluriel est intéressant parce qu'il sous-entend une diffusion encore plus grande que celle du dictionnaire.

6. Je fais évidemment une hypothèse et une interprétation des termes utilisés (et inventés) par Schwitters. Merz est un mot inventé. Allié à Bau (construire, ou construction) il crée une sorte de cathédrale poétique qui donne Merzbau et qui, plastiquement parlant, devient l'antre même de l'œuvre de Schwitters. De Merz je glisse vers Schmerz (douleur), ce qui permet l'interprétation de ce qu'a ressenti l'artiste au moment de la disparition de son œuvre, comme si le nom anticipait son devenir. Signalons aussi qu'il a recommencé son grand œuvre (dont il n'y a aucune trace) à deux reprises, attestant de la référence à ce dernier. Kommerz (commerce) devient le glissement du sens de commercer avec ses contemporains, en inventant une forme artistique totalement nouvelle (anticipant sur l'œuvre in progress, sur l'installation, sur l'inachevé).

7. Jacques Derrida, La vérité en peinture, Paris, Champs Flammarion, 1978, p. 275.

8. Ibid., p. 275-276.

9. Je fais référence à un texte de 2012, Les papiers buvard de la zone, que Jean-Louis Déotte m'avait consacré : «G. Roesz est artiste et universitaire à l'Université de Strasbourg où Lefebvre et les situationnistes d'une part ont influencé plus d'un de ses collègues, puis d'autre part, et surtout, d'éminents déconstructeurs comme Lacoue-Labarthe, Nancy et Payot. Le défi était triple : peuton continuer d'être artiste quand on enseigne l'art, surtout à l'Université ? Peut-on échapper à la critique de la société du spectacle et des simulacres? Peut-on œuvrer sans tracer?» 10. Jacques Derrida, La vérité en peinture, op. cit., p. 276.

\section{RÉSUMÉS}

La présente contribution (rédigée en février-mars 2019) discute les positions sur le numérique défendues par Jean-Louis Déotte dans le numéro 29 de la revue Art Press 2 (2013).

\section{INDEX}

Mots-clés : numérique, représentation, programme, projet, différence 
AUTEURS

GERMAIN ROESZ

Professeur honoraire, Université de Strasbourg, Accra, UR 3402 ; roesz@unistra.fr 